\title{
CONDITIONS FOR A MATRIX TO COMMUTE WITH ITS INTEGRAL
}

\author{
IRVING J. EPSTEIN
}

1. Introduction. Let $U(t)$ be an $n \times n$ matrix whose elements are continuous functions of a parameter $t$. We shall find necessary and sufficient conditions for the relation

$$
U(t) \int_{0}^{t} U(s) d s=\left(\int_{0}^{t} U(s) d s\right) U(t)
$$

to hold in an interval $0 \leqq t \leqq t_{0}$, where $t_{0}$ is so small that throughout the interval $\left[0, t_{0}\right]$ the Jordan canonical form of $U(t)$ has the same form. That is, its off-diagonal elements do not change in the interval.

Matrices $U(t)$ satisfying (1.1) are of interest for various reasons; see, for instance, $[1$, p. 278]. We may mention two occasions where (1.1) occurs. Firstly, consider a system of $n$ homogeneous linear differential equations of the first order for $n$ unknown functions with $U(t)$ as the matrix of coefficients. If we consider the unknown functions as components of a vector, and if we form a matrix $Y$, the $n$ columns of which are $n$ linearly independent solutions of our system, then we have for $Y=Y(t)$ :

$$
\dot{Y}=U Y, \quad Y(0)=I,
$$

where a dot denotes the derivative with respect to $t$ and where $I$ denotes a nonsingular matrix which we may choose to be the unit matrix. If (1.1) holds, then (1.2) can be solved in terms of quadratures. In fact, we have

$$
Y=\exp \int_{0}^{t} U(s) d s .
$$

Secondly, consider the following problem in the theory of systems with periodic coefficients. Let $W(t)$ be an $n \times n$ matrix depending continuously on $t$ such that, for a constant $\omega$,

$$
W(t+\omega)=W(t),
$$

and also

$$
W(-t)=-W(t) .
$$

Then it has been shown in special cases by Demidovic [2] and, more

Received by the editors February 9, 1962. 
generally, by the author [3] that a matrix $Z_{0}(t)$ satisfying

$$
\dot{Z}_{0}=W Z_{0}, \quad Z_{0}(0)=I,
$$

is periodic with period $\omega$, i.e., $Z_{0}(t+\omega)=Z_{0}(t)$. The matrices $W(t)$ form a linear space under addition.

We ask whether we can extend this linear space such that a system of the type (1.6) still will have periodic solutions. A partial answer to this question is given by the following remark: Let $W$ be a fixed matrix satisfying (1.4) and (1.5). Let $E(t)$ be such that

$$
E(t+\omega)=E(t), \quad E(-t)=E(t) .
$$

Then the system

$$
\dot{Z}=(W+\epsilon E) Z, \quad Z(0)=I
$$

will have solutions with the property

$$
Z(t+\omega)=Z(t)
$$

for all values of $\epsilon$ if the matrix $A(t)$ defined by

$$
A(t)=Z_{0}^{-1}(t) E(t) Z_{0}(t)
$$

commutes with its integral. The proof is based on the standard procedure of expanding $Z(t)$ in a power series in $\epsilon$.

2. Matrices commuting with their derivatives. Instead of $U(t)$ we introduce

$$
V(t)=\int_{0}^{t} U(s) d s
$$

and assume that

$$
\dot{V} V-V \dot{V}=0 .
$$

Consider an interval $\left(t_{1}, t_{2}\right)$ such that, for $t_{1} \leqq t \leqq t_{2}$, there exists a differentiable nonsingular matrix $P(t)$ such that

$$
V(t)=P^{-1}(t) J(t) P(t),
$$

where $J(t)$ is in Jordan canonical form. This means that

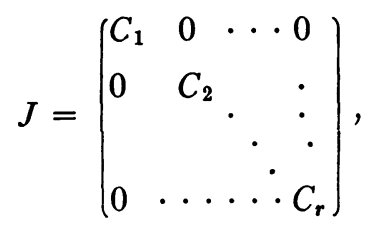

where the submatrices $C_{\rho}(t), \rho=1, \cdots, r$, are $n_{\rho} \times n_{\rho}$ matrices of the form 


$$
C_{\rho}=\alpha_{\rho}(t) I_{\rho}+\delta_{\rho} E_{\rho} .
$$

Here $\alpha_{\rho}(t)$ is a differentiable function of $t, I_{\rho}$ is the $n_{\rho} \times n_{\rho}$ unit matrix, $\delta_{\rho}$ is 0 or 1 , and $E_{\rho}$ is the $n_{\rho} \times n_{\rho}$ matrix with elements

$$
e_{v, \mu}, \quad v, \mu=1, \cdots, n_{\rho},
$$

and

$$
e_{v, v+1}=1, \quad e_{v, \mu}=0 \quad \text { for } \mu-v \neq 1 .
$$

We shall assume that the interval $\left(t_{1}, t_{2}\right)$ is such that no difference $\alpha_{\rho}-\alpha_{\sigma}$ vanishes in a subinterval unless it vanishes identically.

We may assume that, if $\alpha_{\rho}-\alpha_{\sigma}$ vanishes identically, for $\rho \neq \sigma$, either $\delta_{\rho} \neq 0$ or $\delta_{\sigma} \neq 0$. Otherwise, we could contract $C_{\rho}$ and $C_{\sigma}$ into a single diagonal matrix.

Now we have:

Theorem 1. The general $n \times n$ matrix $V(t)$ satisfying (2.1) and having a Jordan canonical form determined by (2.3), (2.4) with constant $n_{\rho}, \delta_{\rho}$ for $t_{1} \leqq t \leqq t_{2}$ is obtained by finding all $n \times n$ matrices $X$ satisfying

$$
J(X J-J X)-(X J-J X) J=0,
$$

determining the nonsingular solutions $P(t)$ of the matrix differential equation

$$
\dot{P}=X P,
$$

and forming

$$
V=P^{-1} J P \text {. }
$$

The matrices $X$ form a linear space (under addition) which depends only on the $n_{\rho}, \delta_{\rho}$, and the set of pairs of subscripts $(\rho, \sigma)$ for which $\alpha_{\rho}-\alpha_{\sigma}$ vanishes identically.

Proof. We observe that, trivially,

$$
J J=J \dot{J}
$$

By differentiating (2.2), we find

$$
\dot{V}=P^{-1} J P+P^{-1} J P+P^{-1} J P .
$$

Because of $P^{-1} P=I$ we have

$$
\dot{P}^{-1} P+P^{-1} P=0, \quad \dot{P}^{-1}=-P^{-1} P P^{-1},
$$

and therefore from (2.2), (2.8), with $X=\dot{P} P^{-1}$ :

$$
\dot{V} V-V \dot{V}=P^{-1}\{-X J+J+J X\} J P-P^{-1} J\{-X J+J+J X\} P=0 .
$$


If we multiply this last equation by $P$ on the left and $P^{-1}$ on the right and then make use of (2.7) we get (2.6). We note that the solutions $X$ of (2.6) form a linear space. In the next section, we shall determine a basis for the linear space of the matrices $X$ and, incidentally, shall also prove that this space does not depend on the functions $\alpha_{\rho}(t)$ but merely on the discrete parameters mentioned in Theorem 1.

Corollary. A system of linear differential equations which, in matrix form, can be written as

$$
\dot{Y}=U Y
$$

where the coefficient matrix $U=\dot{V}$ has the property $U V=V U$, can always be transformed into a system

$$
\dot{Z}=(X+\dot{J}+J X-X J) Z,
$$

where $X, J$ are defined as in Theorem 1. The transformation to be used is, of course, $Z=P Y$, where $P$ is defined as in Theorem 1.

3. The space of matrices $X$. The solutions $X$ of (2.6) may be written as matrices which are composed of submatrices $X_{\rho, \sigma}, \rho, \sigma=1, \cdots, r$, where $X_{\rho, \sigma}$ is a matrix with $n_{\rho}$ rows and $n_{\sigma}$ columns and

$$
X=\left(X_{\rho, \sigma}\right)
$$

with the natural arrangement of the submatrices. From (2.6) we find the equations

$$
C_{\rho}^{2} X_{\rho \sigma}+X_{\rho \sigma} C_{\sigma}^{2}-2 C_{\rho} X_{\rho \sigma} C_{\sigma}=0
$$

where $C_{\rho}$ is given by equation (2.4).

If we let $x_{k l}$ denote the element in the $k$ th row and $l$ th column of $X_{\rho \sigma}$ then (3.2) gives us the scalar equations

$$
\begin{aligned}
\left(\alpha_{\rho}-\alpha_{\sigma}\right)^{2} x_{k, l}+2 \delta_{\rho}\left(\alpha_{\rho}-\alpha_{\sigma}\right) x_{k+1, l}+2 \delta_{\sigma}\left(\alpha_{\sigma}-\alpha_{\rho}\right) x_{k, l+1} \\
+\delta_{\rho}^{2} x_{k+2, l}+\delta_{\sigma}^{2} x_{k, l-2}-2 \delta_{\rho} \delta_{\sigma} x_{k+1, l-1}=0
\end{aligned}
$$

where

$$
k=1,2, \cdots, n_{\rho}, \quad l=1,2, \cdots, n_{\sigma},
$$

and where we define $x_{p q}=0$ if $p>n_{p}$ or $q<1$. Equations (3.3) have to be analyzed for various cases. We may summarize the results as follows:

THEOREM 2. The matrix $X_{\rho \sigma}$ has one of the following structures:

CASE 1. $\alpha_{\rho}-\alpha_{0}$ does not vanish identically (and, therefore, not in any subinterval of $\left(t_{1}, t_{2}\right)$. Then $X_{\rho \sigma}$ is identically zero. 
CASE 2. $\alpha_{\rho}-\alpha_{\sigma} \equiv 0, \delta_{\rho}=0, \delta_{\sigma}=1$. Then the last two columns of $X_{\rho, \sigma}$ are arbitrary, but all other elements of $X_{\rho, \sigma}$ vanish identically.

CASE 3. $\alpha_{\rho}-\alpha_{\sigma} \equiv 0, \delta_{\rho}=1, \delta_{\sigma}=0$. Then the first two rows of $X_{\rho, \sigma}$ are arbitrary but all other elements of $X_{\rho, \sigma}$ vanish identically.

CASE 4. $\alpha_{\rho}-\alpha_{\sigma} \equiv 0, \delta_{\rho}=\delta_{\sigma}=0$. Then we may assume $\rho=\sigma$ (see remarks before Theorem 1), and $X_{\rho \rho}$ is arbitrary.

CASE 5. $\alpha_{\rho}-\alpha_{\sigma} \equiv 0, \delta_{\rho}=\delta_{\sigma}=1$. Denoting the elements of $X_{\rho, \sigma}$ by $x_{l, k}$, where $l=1, \cdots, n_{\rho}$ and $k=1, \cdots, n_{\sigma}$, and if $n_{\rho}>n_{\sigma}$, then the first two rows of $X_{\rho, \sigma}$ are arbitrary and $X_{\rho \sigma}$ has the appearance indicated below:

$$
\left(\begin{array}{cccccc}
x_{11} & x_{12} & x_{13} & x_{14} & x_{15}, & \ldots \\
x_{21} & x_{22} & x_{23} & x_{24} & x_{25}, & \ldots \\
0 & 2 x_{21} & 2 x_{22}-x_{11} & 2 x_{23}-x_{12} & 2 x_{24}-x_{13}, & \ldots \\
0 & 0 & 3 x_{21} & 3 x_{22}-2 x_{11} & 3 x_{23}-2 x_{12}, \ldots \\
0 & 0 & 0 & 4 x_{21} & 4 x_{22}-3 x_{11}, \ldots \\
0 & 0 & 0 & 0 & 5 x_{21}, & \ldots \\
\ldots & \ldots & \ldots & \ldots & \ldots & \ldots
\end{array}\right)
$$

If $n_{\rho}<n_{\sigma}$, the role of rows and columns has to be exchanged, and if $n_{\rho}=n_{\sigma}$, the $X_{\rho, \rho}$ is triangular, but the same shape as above, except that $x_{21}=0$.

Only Case 5 requires a more detailed analysis. However, once the explicit form of $X$ stated above is known, it can be verified with a moderate amount of calculations which will be omitted here.

Acknowledgment. I acknowledge with pleasure the advice given me by Professor W. Magnus of New York University in the preparation of this paper.

\section{REFERENCES}

1. V. V. Nemytskii and V. V. Stepanov, Qualitative theory of differential equations, Princeton Univ. Press, Princeton, N. J., 1960.

2. B. P. Demidovic, On some properties of the characteristic exponents of a system of ordinary linear differential equations with periodic coefficients, Moskov. Gos. Univ. Učen. Zap. 163 Mat. 6 (1952), 123-132. (Russian)

3. I. J. Epstein, Periodic solutions of systems of differential equations, Proc. Amer. Math. Soc. 13 (1962), 690-694.

4. W. Magnus, On the exponential solution of differential equations for a linear operator, Comm. Pure Appl. Math. 7 (1954), 664.

5. M. J. Hellman, Lie algebras arising from systems of linear differential equations, Res. Rep. No. BR-10, New York University, Institute of Mathematical Sciences. Division of Electromagnetic Research.

Evans Signal Laboratory, Ft. Monmouth 